\title{
PERCEPÇÃO DO CUMPRIMENTO DA MISSÃO ORGANIZACIONAL E DESEMPENHO INDIVIDUAL EM UMA EMPRESA PÚBLICA BRASILEIRA
}

\section{Perception of the organizational mission accomplishment and individual performance in a brazilian public company}

Uerlei Valdomiro Araujo

E-mail: uerlei.araujo.uva@gmail.com

Mestre em Gestão Pública pela Universidade Federal do Espírito Santo;

Especialista Lato Sensu em Políticas Públicas em Gênero e Raça pela Universidade Federal do

Espírito Santo; Agente de Correios na Empresa Brasileira de Correios e Telégrafos. Endereço para contato: Rua Jequié, 60, Barcelona, 29166-051, Serra, Espírito Santo, Brasil.

https://orcid.org/0000-0002-2769-014X

Roquemar de Lima Baldam

E-mail: roquemar.baldam@ifes.edu.br

PhD em Engenharia da Produção pela Universidade Federal do Rio de Janeiro; Doutor em Engenharia da produção pela Universidade Federal do Rio de Janeiro; Professor Permanente no

Programa de Gestão Pública da Universidade Federal do Espírito Santo. https://orcid.org/0000-0002-8100-2862

Thalmo de Paiva Coelho Junior E-mail: thalmo@ifes.edu.br

PhD em Engenharia da Produção pelo Instituto Alberto Luiz Coimbra de Pós-graduação e Pesquisa de Engenharia; Doutor em Engenharia da Produção pela Universidade Federal do Rio de Janeiro;

Professor no Instituto Federal de Educação, Ciência e Tecnologia do Espírito Santo; Professor

Permanente no Programa de Gestão Pública da Universidade Federal do Espírito Santo. https://orcid.org/0000-0002-3716-1882

Lourenço Costa

E-mail: lourenco.costa@ifes.edu.br

$\mathrm{PhD}$ em Engenharia da Produção pela Universidade Federal do Rio de Janeiro; Doutor em Engenharia da Produção pela Universidade Federal do Rio de Janeiro; Professor no Instituto Federal de Educação, Ciência e Tecnologia do Espírito Santo; Professor Permanente no Programa de Gestão

Pública da Universidade Federal do Espírito Santo. https://orcid.org/0000-0001-8817-7449

Artigo recebido em 12 de novembro de 2018. Aceito em 5 de agosto de 2019. 


\section{Resumo}

Este artigo aborda o desempenho individual e a percepção do cumprimento da missão organizacional pelos trabalhadores de uma estatal brasileira. Trata-se de um estudo descritivo-exploratório com a aplicação de uma survey utilizando a Escala de Likert para medir o grau de concordância com as afirmativas propostas. Baseando-se em um estudo já realizado em 2017 por Lim, Wang, e Lee com as agências públicas federais dos Estados Unidos, este artigo propõe esclarecimentos sobre o impacto das práticas de gestão de pessoas, como recrutamento e seleção, treinamento, avaliação e recompensa, na percepção do cumprimento da missão organizacional pelos trabalhadores públicos brasileiros, e a respeito de quais os reflexos no desempenho individual. Este estudo tem implicações positivas para os gestores públicos no Brasil, pois possui um olhar singular para as características do serviço público brasileiro, com foco na gestão estratégica de recursos humanos e na gestão do desempenho. Para a análise dos dados coletados se utilizou método quantitativo de regressão linear múltiplo, pesquisa documental, observação direta e revisão de literatura sobre o tema proposto.

Palavras-chave: Gestão de pessoas. Cumprimento da missão organizacional. Gestão do desempenho.

\section{Abstract}

This article addresses the individual performance and the perception of the accomplishment of the organizational mission by the workers of a Brazilian state-owned company. It is a descriptive-exploratory study with the application of a survey using the Likert Scale to measure the degree of agreement with the proposed statements. Based on a study already conducted in 2017 with the United States federal public agencies by Lim, Wang, and Lee, this article proposes clarification on the impact of people management practices such as recruitment and selection, training, evaluation and reward, in the perception of the accomplishment of the organizational mission by the Brazilian public workers and what the reflexes in the individual performance. This study has positive implications for public managers in Brazil, because it has a singular look at the characteristics of the Brazilian public service, focusing on the strategic management of human resources and performance management. For the analysis of the data collected, we used a quantitative method of multiple linear regression, documentary research, direct observation and a literature review on the proposed theme.

Keywords: Human resource management. Organizational mission accomplishment. Performance management.

\section{INTRODUÇÃO}

A Gestão Estratégica de Recursos Humanos (GERH) possibilita identificar e mapear obstáculos para o crescimento da empresa e ajuda a racionalizar tomadas de decisão por parte de seus gestores. De acordo com Dutra (2002), o governo considera como estratégicopara se tornar um modelo gerencial controlador de resultados, em vez de um modelo burocrático controlador de processos-capacitar seus servidores, valorizar a função pública e realizar avaliações de desempenho regulares. Akim e Mergulhão (2015) afirmam que a 
gestão de pessoas como estratégia para o aumento do desempenho é um campo emergente que necessita ser pesquisado.

Klein e Mascarenhas (2016), ao estudarem motivação, satisfação profissional e evasão no serviço público, relacionando satisfação, metas, esforço, desempenho e recompensas, explicam que as recompensas externas, quando atreladas a desempenhos, podem gerar satisfação nos empregados. Segundo os autores, um fator extrínseco, quando associado aos desafios de superar uma meta e de atingir elevado desempenho, pode gerar sensação de realização, promovendo, assim, motivação intrínseca. Depreende-se desta análise que a combinação eficaz de fatores motivacionais extrínsecos e intrínsecos tem o potencial de promover satisfação e aumentar o desempenho e a produtividade individual do empregado (Klein \& Mascarenhas, 2016).

Para Lim, Wang, e Lee (2017), motivação pode ser definida como uma variável dependente de outras variáveis, como treinamento, recrutamento e seleção, avaliação, recompensa e liderança transformadora, podendo influenciar substancialmente - desempenho dos empregados que é esperado pela organização. Apesar de ser constatada durante a pesquisa como uma variável de alta significância, não se explorou quantitativamente ou qualitativamente "motivação", inferindo-se, assim, que este estudo não é absoluto e deixa esta lacuna para novas pesquisas com o objetivo de melhor subsidiar os gestores públicos em tomadas de decisão na gestão de pessoas do serviço público brasileiro. Para melhor elucidação sobre motivação e satisfação e os impactos no desempenho individual no serviço público brasileiro, sugere-se a leitura do estudo promovido por Klein e Mascarenhas (2016).

Algumas abordagens, como a de Bedarkar e Pandita (2014), tratam a motivação como um construto multidimensional que inclui processos cognitivos e afetivos, metas e objetivos, resultados como consequências das ações do indivíduo e suas expectativas de sucesso no alcance de objetivos e resultados almejados.

Para atingir o objetivo proposto de analisar o impacto das práticas de gestão de pessoas na percepção do cumprimento da missão organizacional pelos trabalhadores de uma organização pública brasileira e sua relação com o desempenho individual, baseando-se na pesquisa já realizada com as agências federais dos Estados Unidos, seria prioritário identificar: quais as semelhanças e diferenças do funcionalismo público brasileiro com o norte-americano; quais fatores seriam mais relevantes ou menos relevantes para a satisfação do empregado; e de que forma as diferenças culturais e de legislação poderiam influenciar um resultado diferente nesta pesquisa em comparação à pesquisa realizada por Lim et al. (2017).

Para responder às perguntas elencadas, o presente estudo identifica e relaciona as variáveis independentes Treinamento, Recrutamento, Recompensa e Avaliação como práticas de uma gestão estratégica de recursos humanos e, posteriormente, apresenta a relação 
dessas variáveis com a variável dependente Cumprimento da Missão Institucional. Também relaciona outras variáveis de controle como Liderança e Clareza de Objetivos na Avaliação e propõe uma discussão teórica para os problemas encontrados com foco na Gestão do Desempenho (GD) da organização estudada, o que contribui positivamente para a melhoria da gestão pública brasileira e abre uma discussão para várias abordagens e desafios aos pesquisadores das áreas de gestão e serviço público.

Além de analisar as variáveis que, segundo Lim et al. (2017), impactam o cumprimento da missão institucional, para Lugoboni, Cabral, Chirotto, e Zittei (2018) é primordial que as avaliações de desempenho estejam alinhadas com a razão de existência da organização, assegurando que sua missão institucional seja cumprida. Segundo os autores, esse alinhamento permitirá mensurações sobre controle e até averiguar se os dispêndios de recursos estão sendo aplicados de maneira adequada na missão organizacional (Lugoboni et al., 2018).

\section{REFERENCIAL TEÓRICO}

A GERH, como prática adequada de Recrutamento e Seleção, Treinamento, Recompensa e Avaliação, tornou-se um tema de investigação cada vez mais relevante para o setor público (Arellano-Gaut, Demortain, Rouillard, \& Thoenig, 2013), uma vez que as organizações deste setor existem para satisfazer às necessidades de grupos de clientes particulares por meio da maximização do desempenho organizacional, em vez da busca por lucros financeiros apenas. Porém, para uma organização conseguir que todos os seus colaboradores estejam atualizados, capacitados e satisfeitos, ou seja, colocar essa estratégia em prática conforme a necessidade dela, é preciso realizar cursos e/ou treinamentos para atualização contínua, ter um sistema de avaliação de desempenho contínuo e eficiente, administrar recompensas e benefícios e ter sistematizados o recrutamento e a seleção de pessoal para os cargos, funções e atividades. Além de corroborar com a melhora do desempenho dos colaboradores, a capacitação contínua também impacta a motivação, a autoconfiança e a abertura a mudanças (Yu, Dong, Shen, Khalifa, \& Hao, 2013).

Assim, é notório o fato de que as organizações teriam investimentos financeiros consideráveis com as práticas de GERH, sabendo que tais práticas demandam capacitações e implementações de política periodicamente em razão do número de colaboradores das organizações públicas e da rotatividade de pessoal. Essa ideia é reforçada por Currie e Procter (2005), afirmando que quando os recursos externos são limitados, as organizações são forçadas a olhar internamente para as áreas potenciais de atuação, portanto, o desenvolvimento da estratégia em organizações do setor público deve ter em conta os recursos internos e uma série de importantes atores estratégicos. 
Com isso, este estudo mostra como essa ideia de atender a necessidade de uma GERH pode ser desenvolvida e colocada em prática numa organização pública brasileira, apresentando as etapas deste processo e comparando com os resultados de pesquisa semelhante realizada com agências federais dos Estados Unidos por Lim et al. (2017).

Neely, Gregory, e Platts (2005) afirmam que o desempenho de uma empresa é a função entre sua eficiência e sua eficácia. Eles definem Medição do Desempenho como o processo de quantificação da eficiência e da eficácia de uma ação; Medida de Desempenho como a métrica utilizada para quantificar a eficiência e a eficácia de uma ação; e Sistema de Medição de Desempenho como o conjunto de métricas utilizadas para a quantificação da eficiência e da eficácia das ações.

Para Snell, Morris, e Bohlander (2015), a gestão e avaliação do desempenho é o processo de criar um ambiente de trabalho no qual as pessoas podem realizar o melhor de suas habilidades, a fim de alcançar as metas da empresa. De acordo com Taticchi, Balachandran, e Tonelli (2012), a avaliação e a gestão do desempenho funcionam como facilitadores para que as empresas possam estabelecer metas e estratégias para o sucesso, executar atividades tomando decisões que reflitam nos resultados, monitorar os resultados e apontar melhorias a serem realizadas.

De maneira similar, Gruman e Saks (2011) desenvolveram uma pesquisa fundamentando suas propostas em literatura transversal ao tema. Eles apresentaram um modelo de GD no qual o engajamento do empregado seria um ponto-chave para a melhoria do seu desempenho e discutiram elementos que poderiam promover tal engajamento (Gruman \& Saks, 2011).

Portanto, fundamentando-se nas propostas de Gruman e Saks (2011) e nos ensinamentos dos demais autores que fundamentam a presente pesquisa, serão discutidas na quarta seção as teorias que abordam possíveis soluções para problemas elencados na organização estudada, abordando práticas de GERH e GD para a melhoria da percepção do cumprimento da missão organizacional pelos trabalhadores e do desempenho individual.

\section{METODOLOGIA}

$\mathrm{Na}$ coleta de dados utilizou-se a aplicação de um questionário de pesquisa e, para avaliar o grau de concordância do empregado com as afirmativas propostas, utilizou-se Escala Likert para gerar dados numéricos que, posteriormente, geraram dados estatísticos que serão discutidos na quarta seção.

Para a coleta de dados na empresa pública estudada, foram enviados questionários eletrônicos a 21 mil funcionários que possuem cadastro no e-mail corporativo da empresa. 
Desses envios, obteve-se retorno de 2.117 respostas, gerando uma taxa de retorno de $10 \%$ do total de formulários enviados. Ressalta-se que a maioria dos 21 mil funcionários, contatados via e-mail, executam serviços administrativos, gerenciais ou de apoio técnico em unidade operacional, ou seja, caso a pesquisa seja reaplicada entre os funcionários atuantes em setores mais operacionais, onde se executam os serviços que exigem maior esforço físico e manual, é possível que se obtenham resultados diferentes.

Para fins de avaliação da variável dependente Realização da Missão Institucional, com base no estudo original de Lim et al. (2017), foram replicadas as perguntas realizadas naquele estudo mensuradas pela escala de 5 pontos Likert-type (discordo totalmente, discordo, não concordo nem discordo, concordo e concordo totalmente).

Tendo em vista o objetivo proposto por Lim et al. (2017), optou-se por desenvolver abordagem descritiva e exploratória por meio de uma pesquisa quantitativa para avaliar - grau de significância das práticas de GERH (Treinamento, Recrutamento e Seleção, Avaliação e Recompensa) na percepção do cumprimento da missão organizacional pelos trabalhadores de uma organização pública brasileira e o impacto dessas práticas no desempenho dos trabalhadores. Adicionalmente à survey aplicada para a coleta de dados, adotou-se a observação direta, pesquisa documental e uma revisão de literatura para preencher as lacunas não respondidas, após a análise dos dados estatísticos.

Para comparar os resultados da pesquisa realizada com as agências federais dos Estados Unidos por Lim et al. (2017) com os resultados desta, após a aplicação do questionário de pesquisa, a percepção do empregado público, bem como o grau de concordância com as afirmativas relacionadas à GERH e à GD foram representados por dados numéricos. Assim, realizaram-se análises estatísticas com método de regressão linear múltiplo a partir dos dados coletados (Hair, Black, Babin, Anderson, \& Tatham, 2009).

Adicionalmente, após uma revisão de literatura sobre "as práticas de gestão de pessoas e o impacto destas na percepção do cumprimento da missão organizacional pelos trabalhadores", foi realizada a análise descritiva dos dados estatísticos com o auxílio do software Statistical Package for the Social Sciences (SPSS). Essa análise contribuiu para identificar problemas característicos do funcionalismo público brasileiro no que se refere a treinamento, recrutamento e seleção, recompensa e avaliação como práticas da GERH. Por meio de pesquisa bibliográfica foram identificados autores que pesquisaram sobre problemas encontrados em processos de gestão e avaliação de desempenho, satisfação, recrutamento e recompensa, conforme já elencado na segunda seção e discutido na quarta seção deste artigo.

Ressalta-se que este estudo não pretende identificar todas as melhores práticas de GERH ou todos os fatores que exercem influência no desempenho de uma organização, mas identificar aqueles que contribuam para o principal objetivo desta pesquisa, a 
saber: quais as variáveis de maior significância na percepção do cumprimento da missão organizacional pelos trabalhadores públicos brasileiros e qual o impacto dessas práticas no desempenho individual.

Para tanto, considerou-se fundamental a pesquisa literária sobre fatores que impactam negativa ou positivamente o desempenho individual dos empregados em uma organização, os fatores que influenciam na percepção do cumprimento da missão institucional pelos trabalhadores e de que maneira as práticas de GERH, como Treinamento, Recrutamento e Seleção, Recompensa e Avaliação podem contribuir como vantagem competitiva à organização.

Akim e Mergulhão (2015) apresentaram, por meio de pesquisa bibliométrica, um panorama da produção intelectual no campo da medição do desempenho na gestão pública entre 1980 e fevereiro de 2013. Suas bases foram a ISI Web of Science e a Scielo, de forma a refletir as pesquisas internacionais sobre o tema. As palavras-chave na base ISI Web of Science foram: performance measurement, performance measure, performance evaluation, performance assessment, performance appraisal, associadas às palavras-chave da área de gestão pública: public sector, public service, public organization e public management. Essa pesquisa resultou em uma amostra de 268 artigos, entre os quais 48 concentram $68 \%$ das citações.

Desses 48, analisaram-se os 10 mais citados, mas nenhum dos artigos diz respeito à avaliação de desempenho do indivíduo, mas das organizações públicas como um todo, programas públicos, etc. Tal constatação, somada ao fato de os escritos não abordarem a percepção do trabalhador e sua relação com o trabalho, motivou a utilização de base diversa da utilizada pelos autores para discorrer sobre GD, GERH e o impacto de ambas na percepção do cumprimento da missão institucional pelos trabalhadores.

Considerando que o foco da presente pesquisa é a percepção do empregado, concluiuse que aquelas palavras-chave, utilizadas por Akim e Mergulhão (2015) trouxeram uma amostra pequena de estudos realizados sobre o tema e, portanto, insuficiente para uma discussão com foco na percepção do trabalhador sobre as variáveis Avaliação, Recompensa, Recrutamento e Seleção e Treinamento como práticas de uma GERH, sendo necessário modificar também as palavras-chave para a presente pesquisa.

Com as sugestões de leitura disponibilizadas pela base de dados Scielo e Capes, foi possível acessar outros artigos relacionados ao tema da presente pesquisa e que não faziam parte dos resultados da busca inicial. Além de cuidadosa análise das referências utilizadas por Lim et al. (2017) em sua pesquisa com as agências federais dos Estados Unidos, recorreuse a cruzamento de dados com as palavras-chave, em que se utilizou o software Mendeley para ajudar na busca por referencial teórico sobre o tema proposto. 
Tabela 1

Identificação das variáveis

\begin{tabular}{ll}
\hline \multicolumn{1}{c}{ Variável } & \multicolumn{1}{c}{ Afirmativa/Pergunta } \\
\hline $\begin{array}{l}\text { Realização da missão ins- } \\
\text { titucional (dependente) }\end{array}$ & Minha empresa é muito bem-sucedida no cumprimento de sua missão. \\
\hline $\begin{array}{l}\text { Recompensa (indepen- } \\
\text { dente) }\end{array}$ & $\begin{array}{l}\text { As promoções na minha unidade de trabalho são baseadas em meritocracia. } \\
\text { Meu salário pode variar de acordo com o meu desempenho. }\end{array}$ \\
\hline $\begin{array}{l}\text { Treinamento (indepen- } \\
\text { dente) }\end{array}$ & $\begin{array}{l}\text { Minhas necessidades de treinamento são atendidas. } \\
\text { Quão satisfeito você está com o treinamento que você recebe para realizar o } \\
\text { seu trabalho atual? }\end{array}$ \\
\hline $\begin{array}{l}\text { Recrutamento (indepen- } \\
\text { dente) }\end{array}$ & $\begin{array}{l}\text { Minha unidade de trabalho está preparada para recrutar pessoas com as habi- } \\
\text { lidades necessárias à área. }\end{array}$ \\
\hline $\begin{array}{l}\text { Avaliação (independente) } \\
\text { Minha avaliação de desempenho é um reflexo justo do meu desempenho. } \\
\text { Nazer para ser avaliado em níveis diferentes de desempenho. }\end{array}$ \\
$\begin{array}{l}\text { Em minha organização me sinto encorajado a apresentar novas e melhores } \\
\text { formas de realizar o trabalho proposto pela gestão. }\end{array}$ \\
$\begin{array}{l}\text { Em minha organização os funcionários têm autonomia com relação aos pro- } \\
\text { cessos de trabalho, sem a necessidade de serem gerenciados o tempo todo em } \\
\text { tomadas de decisões menos complexas. } \\
\text { Em minha organização os líderes inspiram altos níveis de compromisso e moti- } \\
\text { vação. } \\
\text { Os líderes de minha organização mantêm elevados padrões de honestidade e } \\
\text { integridade. }\end{array}$ \\
$\begin{array}{l}\text { Eu sei como e quanto o meu trabalho se relaciona com os objetivos e priorida- } \\
\text { des da organização. }\end{array}$ \\
\hline controle)
\end{tabular}

O levantamento das variáveis independentes, dependente e de controle foi efetuado com base na pesquisa de Lim et al. (2017), e, durante a construção do questionário, considerouse relevante o levantamento de algumas hipóteses, como:

1. A estabilidade do funcionalismo público brasileiro faz com que a recompensa não tenha alta significância na mudança de comportamento do empregado;

2. O impedimento legal de remuneração variável para o funcionalismo público brasileiro faz com que fatores externos tenham menos influência na satisfação do empregado em detrimento de fatores intrínsecos ao indivíduo, tendo em vista que a carreira pública, os proventos e vantagens já são garantias previstas em lei;

3. A estabilidade do empregado público brasileiro pode influenciar sua percepção com relação à avaliação, ao seu desempenho e à necessidade de melhoria;

4. As práticas de recrutamento e seleção são pouco percebidas pelo empregado público, tendo em vista que o ingresso na carreira pública brasileira é via concurso público e a ascensão na carreira tem critérios previamente definidos pela administração pública e por acordos coletivos, no caso de empresas estatais. 


\section{RESULTADOS E DISCUSSÕES}

As afirmativas elencadas na Tabela 1 foram utilizadas com o intuito de verificar a relação da variável dependente com as seguintes variáveis independentes, medidas na pesquisa:

a) Recompensa: ou seja, como as recompensas no ambiente de trabalho funcionam na organização estudada;

b) Treinamento: se as necessidades de treinamento dos funcionários são atendidas;

c) Recrutamento: se a organização recruta adequadamente os colaboradores;

d) Avaliação: se os funcionários são adequadamente avaliados por seu desempenho.

Além dessas variáveis independentes, foram acrescentadas duas variáveis que, no artigo original, funcionaram como variáveis de controle, mas que surpreenderam quando aplicadas à organização brasileira em análise, sendo elas:

a) Liderança, sendo medido o impacto de vários aspectos da atuação dos líderes junto às equipes;

b) Clareza de objetivos, avaliando se os colaboradores têm clara a relação de seu trabalho com os objetivos da organização.

No estudo, as variáveis Recompensa, Treinamento, Avaliação e Liderança eram compostas de mais de uma questão, sendo cabível, portanto, a realização da avaliação da confiabilidade do grupo de perguntas para aferir a variável em questão.

Tabela 2

Confiabilidade das variáveis

\begin{tabular}{ccc}
\hline \multicolumn{3}{c}{ Estatísticas de confiabilidade } \\
\hline Variável & Alfa de Cronbach & N. de questões \\
\hline Recompensa &, 546 & 2 \\
Treinamento &, 907 & 2 \\
Avaliação &, 810 & 2 \\
Liderança &, 823 & 4 \\
\hline
\end{tabular}

Segundo Hair et al. (2009), para que uma variável seja devidamente mensurada por duas ou mais perguntas, é necessário que o Alfa de Cronbach, calculado entre essas perguntas, seja superior a 0,7. Conforme se verifica na Tabela 2, todos os alfas são satisfatórios, exceto 
o da variável recompensa, o que indica que as perguntas sobre a percepção de recompensa são insuficientes para aferir a variável independente sobre a sensação de recompensa. Nesse sentido, é relevante destacar que o serviço público brasileiro, por questões legais, não permite a remuneração variável como ocorre em outros países, e isso pode dificultar a correta aferição da percepção dos trabalhadores públicos para essa variável.

Assim, inferiu-se que as hipóteses 1 e 2, levantadas na terceira seção, são relevantes e fazem todo o sentido para a análise do caso brasileiro. Para Klein e Mascarenhas (2016), diversos outros fatores intrínsecos e extrínsecos também afetam a percepção e o desempenho do funcionário público em sua atuação. Entre eles, pode-se citar na dimensão intrínseca ou simbólica do trabalho o altruísmo, o prazer com o trabalho, o comprometimento, o senso de dever, a autonomia e a responsabilidade de servir à sociedade. Já os fatores extrínsecos, como salário e benefícios, seriam menos importantes para o servidor público em comparação com o privado (Klein \& Mascarenhas, 2016).

Segundo Campbell e $\operatorname{Im}$ (2016), motivação no serviço público tem completa ligação com o atingimento dos valores e objetivos da organização pública, porém nem todos os empregados contribuem com desempenho igual, nem reconhecem igualmente seu papel no cumprimento da missão organizacional. Muitas vezes, funcionários de alto desempenho que teriam grande impacto positivo na empresa se sentem desmotivados e acabam não contribuindo plenamente para o cumprimento da missão organizacional. Em muitos casos, isso ocorre em razão do fato de, no Brasil, os funcionários públicos possuírem estabilidade e seus salários serem estáticos com aumentos publicados por decreto/lei após a aprovação dos legislativos ou após Acordo Coletivo de Trabalho (ACT), no caso das empresas estatais.

Dessa forma, a maneira como os trabalhadores públicos brasileiros desempenham suas atividades tem pouca ou nenhuma influência sobre os seus aumentos salariais, $e$ isso justifica a confiabilidade baixa para a variável Recompensa. Outro motivo é que no modelo de contratação da empresa estatal estudada é obrigatória a abertura de concurso público $e$ isso impede uma análise de perfil com foco nas atividades a serem exercidas após a contratação. Também é importante destacar que a empresa estudada é $100 \%$ estatal e por isso sofre muita influência de atores políticos em sua gestão, ocasionando muitas promoções por indicação e não por mérito ou carreira, o que pode significativamente influenciar os resultados da variável Recrutamento e Seleção, ao contrário do que ocorre com os trabalhadores públicos norte-americanos. Essa análise justifica a hipótese 4 elencada na terceira seção.

Assim, evidenciam-se diferenças relevantes entre o modelo apresentado por Lim et al. (2017) e a realidade de uma empresa pública brasileira, cujo padrão de funcionamento seja mais assemelhado às características do serviço público brasileiro. 
$\mathrm{Na}$ análise de dados desta pesquisa, outro fator interessante foi o fato de a variável Liderança, que para Lim et al. (2017) funcionava apenas como variável de controle, neste estudo teve o maior índice de significância entre todas as variáveis verificadas, ou seja, apesar de inicialmente não ser hipótese de influência na variável dependente, no caso brasileiro foi a mais relevante entre as variáveis estudadas.

Tabela 3

Coeficientes $^{a}$

\begin{tabular}{|c|c|c|c|c|c|c|c|c|}
\hline \multirow{2}{*}{\multicolumn{2}{|c|}{$\begin{array}{c}\text { Modelo } \\
\text { B }\end{array}$}} & \multicolumn{2}{|c|}{$\begin{array}{c}\text { Coeficientes não } \\
\text { padronizados }\end{array}$} & \multirow[t]{2}{*}{$\begin{array}{c}\text { Coeficientes } \\
\text { padronizados }\end{array}$} & \multirow{2}{*}{$T$} & \multirow{2}{*}{$\begin{array}{l}\text { Sig. } \\
\text { Tolerân- } \\
\text { cia }\end{array}$} & \multicolumn{2}{|c|}{$\begin{array}{l}\text { Estatísticas de } \\
\text { colinearidade }\end{array}$} \\
\hline & & $\begin{array}{l}\text { Erro-pa- } \\
\text { drão }\end{array}$ & Beta & & & & VIF & \\
\hline \multirow{2}{*}{1} & (Constante) & 2,010 &, 057 & & 35,546 & ,000 & & \\
\hline & LIDER-M & ,459 & ,018 & ,481 & 25,259 & ,000 & 1,000 & 1,000 \\
\hline \multirow{3}{*}{2} & (Constante) & 1,790 & , 058 & & 30,950 & ,000 & & \\
\hline & LIDER-M & ,318 & 021 &, 334 & 14,989 & ,000 & 686 & 1,457 \\
\hline & TREIN-M & ,222 & ,019 & ,263 & 11,809 & ,000 & 686 & 1,457 \\
\hline \multirow{4}{*}{3} & (Constante) & 1,476 & , 073 & & 20,130 & ,000 & & \\
\hline & LIDER-M & 267 &, 022 & ,280 & 11,975 & ,000 & ,609 & 1,641 \\
\hline & TREIN-M & 202 & 019 & 240 & 10,742 & , 000 & ,670 & 1,492 \\
\hline & $\begin{array}{l}\text { CLAREZA DE OBJE- } \\
\text { TIVOS-M }\end{array}$ & 133 & ,019 & , 144 & 6,873 & ,000 & ,764 & 1,309 \\
\hline \multirow{5}{*}{4} & (Constante) & 1,462 & , 073 & & 20,047 & ,000 & & \\
\hline & LIDER-M & ,232 &, 023 & ,244 & 10,009 & ,000 &, 556 & 1,799 \\
\hline & TREIN-M & 167 & ,020 & 198 & 8,371 & ,000 & ,589 & 1,699 \\
\hline & $\begin{array}{l}\text { CLAREZA DE OBJE- } \\
\text { TIVOS-M }\end{array}$ & , 123 & ,019 & 133 & 6,381 & ,000 & ,756 & 1,322 \\
\hline & RECRUT-M & , 090 & 018 & 118 & 5,027 & ,000 & ,602 & 1,662 \\
\hline \multirow{6}{*}{5} & (Constante) & 1,449 & , 073 & & 19,937 & ,000 & & \\
\hline & LIDER-M & 199 &, 024 & 209 & 8,140 & , 000 & 498 & 2,007 \\
\hline & TREIN-M & 152 & ,020 & 180 & 7,499 & ,000 & ,570 & 1,756 \\
\hline & $\begin{array}{l}\text { CLAREZA DE OBJE- } \\
\text { TIVOS-M }\end{array}$ & , 122 & ,019 & 132 & 6,346 & ,000 & ,756 & 1,322 \\
\hline & RECRUT-M & , 079 & ,018 & , 103 & 4,345 & ,000 &, 588 & 1,701 \\
\hline & RECOMP-M & ,088 & 021 & , 097 & 4,267 & ,000 & 629 & 1,589 \\
\hline \multirow{7}{*}{6} & (Constante) & 1,412 & , 074 & & 19,057 & , 000 & & \\
\hline & LIDER-M & 183 &, 025 & 192 & 7,297 & ,000 & ,470 & 2,130 \\
\hline & TREIN-M & , 145 & , 020 & 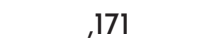 & 7,098 &, 000 & ,559 & 1,787 \\
\hline & $\begin{array}{l}\text { CLAREZA DE OBJE- } \\
\text { TIVOS-M }\end{array}$ & , 114 & ,020 & , 123 & 5,830 & ,000 & ,735 & 1,361 \\
\hline & RECRUT-M & ,072 & ,018 & ,094 & 3,927 & ,000 &, 575 & 1,738 \\
\hline & RECOMP-M & ,085 &, 021 & ,095 & 4,146 & ,000 & ,628 & 1,592 \\
\hline & AVAL-M & ,048 & 019 &, 059 & 2,572 & , 010 & ,630 & 1,587 \\
\hline
\end{tabular}

Nota. a. Variável Dependente: MISSAO. 
Uma causa provável desse resultado é o fato de as nomeações da maioria das funções de liderança do serviço público brasileiro advirem da influência de atores políticos e não por mérito necessariamente. Essa prática normalmente torna as gerências pontos de tensão dentro da organização, o que gera uma expectativa de lideranças despreparadas para os trabalhadores da organização. Assim, lideranças bem-preparadas e que inspiram confiança nos colaboradores acabaram obtendo um papel mais significante do que se esperava em comparação com o estudo original.

Nesse caso, inferiu-se que a hipótese 3, levantada na terceira seção, não tem sustentação plausível nos resultados, pois a estabilidade não afetou a percepção do trabalhador necessariamente, mas a liderança foi um fator de sucesso para que o trabalhador percebesse a necessidade de melhoria com foco no desempenho.

Oliveira Nogueira, Costa, e Santos Claro (2015), ao estudarem os estilos de liderança e a relação destes com o comprometimento organizacional afetivo concluíram que os estilos de liderança não são capazes de alterar a percepção do trabalhador. Segundo os autores, existem outras variáveis que mais impactam a percepção dos empregados. Embora os líderes tenham papel fundamental na gestão do desempenho das equipes e consequentemente nos resultados apresentados por elas, pouco influenciam a percepção do empregado em aspectos cognitivos e afetivos à organização (Oliveira Nogueira et al., 2015).

Muitos estudos recentes têm considerado a influência do estilo de liderança e seus efeitos positivos no atingimento dos objetivos organizacionais em um contexto público. Segundo Bronkhorst, Steijn, e Vermeeren (2015), líderes são capazes de indicar com clareza os objetivos da empresa, motivar os empregados ao atingimento da missão e melhorar a percepção do empregado com relação à importância do seu papel para o alcance dos objetivos e metas da organização. Além disso, de acordo com Bronkhorst et al., outros fatores são determinantes para um líder inspirador, como demonstrar consideração individualizada, ser fonte de admiração, estimular os funcionários a ver novas perspectivas e promover motivação. 
Tabela 4

Resumo do modelog

\begin{tabular}{ccccc}
\hline Modelo & $\mathbf{R}$ & $\mathbf{R}$ quadrado & $\mathbf{R}$ quadrado ajustado & Erro padrão da estimativa \\
\hline 1 &, $481^{\mathrm{a}}$ &, 232 &, 231 &, 80022 \\
2 &, $528^{\mathrm{b}}$ &, 279 &, 279 &, 77525 \\
3 &, $543^{\mathrm{c}}$ &, 295 &, 294 &, 76690 \\
4 &, $551^{\mathrm{d}}$ &, 303 &, 302 &, 76254 \\
5 &, $556^{\mathrm{e}}$ &, 309 &, 308 &, 75945 \\
6 &, $558^{\mathrm{f}}$ &, 311 &, 310 &, 75844 \\
\hline
\end{tabular}

Nota. a. Preditores: (Constante), LIDERM;

b. Preditores: (Constante), LIDERM, TREINM;

c. Preditores: (Constante), LIDERM, TREINM, VISAO;

d. Preditores: (Constante), LIDERM, TREINM, VISAO, RECRUT;

e. Preditores: (Constante), LIDERM, TREINM, VISAO, RECRUT, RECOMPM;

f. Preditores: (Constante), LIDERM, TREINM, VISAO, RECRUT, RECOMPM, AVALM;

g. Variável Dependente: MISSAO.

$O R^{2}$ varia entre $\mathrm{O}$ e l, indicando, em percentagem, o quanto o modelo consegue explicar os valores observados (Hair et al., 2009). Dessa forma, o baixo resultado do $R^{2}$ apresenta a necessidade de pesquisar outras variáveis que impactam a percepção do cumprimento da missão institucional pelos trabalhadores públicos da empresa brasileira estudada.

Apesar de todo o cuidado na revisão e da utilização de um estudo já concretizado como referência para esta pesquisa, o $\mathrm{R}^{2}$ obtido no presente estudo foi consideravelmente baixo, mesmo com a utilização de dados primários e ampla pesquisa em nível nacional. Com isso, infere-se que no modelo de organização estudado há variáveis muito importantes não consideradas que têm influência sobre a variável dependente Realização da Missão Institucional, como já elencado anteriormente com o exemplo da variável Motivação.

Além da análise quantitativa das variáveis dependente, independente e de controle, utilizaram-se métodos qualitativos, nos quais, por meio da observação direta e pesquisa documental, foi possível verificar vários pontos em melhoria e preencher algumas lacunas que não foram explicitadas com os resultados obtidos com a survey. Dessa forma, optou-se por realizar uma revisão de literatura para cada situação-problema encontrada na organização estudada durante a pesquisa.

\subsection{INDICADORES E METAS IMPOSTOS AOS EMPREGADOS}

Para Gruman e Saks (2011), as metas devem ser condizentes com os objetivos da organização, porém, quando se permite que os empregados tenham voz no estabelecimento das metas, estas se alinham aos seus interesses e valores, produzindo bem-estar e gerando 
engajamento. Ojokuku e Sajuyigbe (2014), nessa vertente, afirmam que a alta influência da voz do empregado aumenta o potencial de alavancar resultados. Para Leeuw e Van Den Berg (2011), sem uma boa definição e uma boa implementação das métricas de desempenho, é difícil acarretar ganho de produtividade para a organização.

Dessa forma, conclui-se que seria importante empresa e empregados, em conjunto, construírem novos indicadores e metas com foco no negócio da instituição. De acordo com Gruman e Saks (2011), essa medida melhoraria a motivação dos empregados, seu engajamento, e, logo, seu desempenho.

\subsection{NÃO HÁ ACOMPANHAMENTO DOS INDICADORES POR PARTE DOS EMPREGADOS}

Leeuw e Van Den Berg (2011) pesquisaram sobre desempenho organizacional baseado na influência do comportamento dos empregados mediante práticas de gestão e avaliação do desempenho. Quanto aos indicadores de desempenho, os autores relacionam dois aspectos a serem observados: devem proporcionar uma visão balanceada e completa do desempenho dos empregados e dar à direção da empresa uma visão imediata dos gargalos operacionais.

Leeuw e Van Den Berg (2011) destacaram a importância de o empregado acompanhar os indicadores de seu desempenho diariamente para melhorar e permanecer motivado. A falta de informações ao longo do período de avaliação prejudica o desempenho do funcionário, pois este, por desconhecimento de que seu desempenho está aquém do esperado, não altera seu comportamento (Snell et al., 2015).

\subsection{AUSÊNCIAS DE CRITÉRIOS OBJETIVOS NO ESTABELECIMENTO DE METAS E FALTA DE CLAREZA NOS OBJETIVOS}

Taticchi et al. (2012) apontam, como uma das principais intervenções para empresas que desejam aumentar a efetividade de um Sistema de Medição e Gestão do Desempenho (Performance Measurement and Management System (PMM System)), que os modelos PMM incorporem inter-relações lógicas de causa e efeito entre ações e resultados.

Segundo os autores, as empresas têm dificuldades de interpretar informações advindas da medição dos processos operacionais e reconhecem que a tarefa de compreender a cadeia de valor e a relação de causa e efeito nos diversos tipos de organizações é um desafio para esse campo de pesquisa. Dada a complexidade do tema, Neely et al. (2005) incluíram 
em seu trabalho, como agenda de pesquisa, algumas questões relacionadas à medição de desempenho individual do empregado, quais sejam:

a) A medida do desempenho deveria focar no processo, no resultado do processo ou em ambos?

b) Como a medição do desempenho pode ser desenhada de forma a encorajar a cooperação interfuncional?

c) Como a medição do desempenho pode ser desenhada de forma a encorajar um comportamento apropriado?

Desse modo, a necessidade de aumentar a objetividade dos critérios para estabelecimento de metas e clareza nos objetivos abre oportunidades para que sejam realizados estudos adicionais sobre a formação de critérios e construção de indicadores objetivos dentro das diversas áreas da empresa (indicadores específicos e distintos para as áreas administrativas e operacionais, por exemplo).

\subsection{REMUNERAÇÃO VARIÁVEL DE ACORDO COM O DESEMPENHO DA EMPRESA}

Aguinis e Bradley (2015) afirmam que o sistema de remuneração variável por atingimento das metas por equipe desmotiva os empregados de alto desempenho porque não terão seus esforços recompensados, e beneficia os empregados de baixo desempenho porque poderão almejar algum ganho financeiro mesmo sem ter feito por merecer (conhecido como "carona" ou freerider). Nesse caso, os autores sugerem a adoção de um sistema de remuneração variável por desempenho individual (Peformance-Related Pay $(P R P)$ ), em que cada empregado seria premiado segundo sua própria produção acima das metas estabelecidas, mantendo a satisfação e motivação dos colaboradores (Aguinis \& Bradley, 2015).

Apesar de haver limitações impostas pela legislação do próprio setor público brasileiro, estudos indicam a possibilidade de a implantação de sistemas PRP dentro da administração pública ter efeitos positivos (de Assis \& Reis, 2011).

Assis e Reis (2011) afirmam que mensuração baseada nos resultados é mais indicada e adequada ao caso brasileiro em razão da sua objetividade. Por outro lado, Snell et al. (2015) salientam que avaliar a produção de um funcionário em uma linha de produção é relativamente fácil e pode-se realizar de forma objetiva, porém, quando se trata de funcionário da área administrativa, é mais difícil e subjetivo. Esse é, portanto, um ponto a 
ser observado pela administração pública brasileira na discussão com os empregados sobre indicadores e metas a serem utilizados em seus respectivos setores.

Segundo Assis e Reis (2011), para aumentar a possibilidade de sucesso na implementação de um sistema de PRP na administração pública brasileira, deve-se estruturar - modelo acerca de equipes e não de indivíduos; utilizar unidade de inteligência para mensurar, controlar e aperfeiçoar a competição com a cooperação entre os colaboradores; $e$ criar incentivos diferenciados por tipo de tarefa.

\subsection{RECOMPENSAS BASEADAS APENAS NO QUE FOI REALIZADO PELO EMPREGADO}

Aguinis e Bradley (2015) incentivam as empresas a implementarem sistemas de gestão do desempenho em vez de simplesmente aplicarem uma avaliação anual e uma revisão. Para os autores, os gerentes deveriam adotar mecanismos de recompensas por metas específicas, que agregam valor à organização. Dessa forma, tiram o foco da recompensa por ano trabalhado e enfatizam recompensa por ações específicas produzidas pelos empregados, fazendo com que se sintam mais motivados. Essas mudanças deixam de recompensar o que foi realizado para focar no que será produzido.

\subsection{FEEDBACK FALHO}

Constatou-se nos resultados desta pesquisa que há problemas no feed back da avaliação de desempenho ao empregado público. A avaliação de desempenho dos empregados é realizada de seis em seis meses, ou anualmente, dependendo da posição funcional do empregado, departamento ou órgão de governo, e, portanto, trata-se de um procedimento com periodicidade previsível e estático, sem foco no resultado acompanhado pela gestão do processo ao longo do período avaliativo com feedback constante e propositivo de melhorias para o alcance dos resultados pretendidos.

Gabelica, Van Den Bossche, De Maeyer, Segers, e Gijselaers (2014) afirmam que dar o feedback às equipes é uma ferramenta poderosa para incrementar seu aprendizado, seu desempenho, sua satisfação e motivação. Para Ford, Latham, e Lennox (2011), a maioria das empresas opta por fixar um intervalo de tempo para se realizarem as reuniões de feedback sobre o desempenho. Porém, dessa forma, como se pode avaliar o desempenho continuamente se as reuniões são programadas em períodos previamente fixados? Não tem como os gerentes avaliarem todo o período sem se esquecerem de alguma coisa. Entretanto, quando não há previsibilidade da data da avaliação e do feedback, a tendência é que os 
empregados permaneçam engajados durante todo o período (Gabelica et al., 2014; Ford et al., 2011). Nesse cenário conclui-se que os problemas de feedback nas organizações públicas brasileiras seriam minimizados caso adotassem as seguintes medidas:

a) alteração do cronograma de avaliação e feedback do desempenho do empregado, deixando de ser fixo para ter uma programação menos previsível, conforme sugerem Ford et al. (2011) e Gabelica et al. (2014);

b) o feedback deve ser contínuo, ou seja, ao longo do período a ser avaliado, possibilitando que o empregado corrija seu comportamento e seu desempenho antes da avaliação propriamente dita, mantendo-se motivado a colaborar para o atingimento das metas propostas (Snell et al., 2015);

c) os gerentes devem adotar a prática de coaching e mentoring com os empregados (Ford et al., 2011), auxiliando-os na melhoria no desempenho;

d) utilizar os empregados de alto desempenho como coachs e mentors (Aguinis \& Bradley, 2015; Russell, Ferris, Thompson, \& Sikora, 2016).

\subsection{RESPONSABILIDADE DAS AVALIAÇÕES DE DESEMPENHO A CARGO SOMENTE DOS LÍDERES IMEDIATOS}

Diversos autores pesquisados criticaram os sistemas de Gestão do Desempenho e de Gestão de Recursos Humanos nos quais a responsabilidade da avaliação incide apenas sobre o líder imediato do avaliado. Segundo Lotta (2002), esse tipo de modelo de avaliação restringe a visão com relação ao avaliado e pode haver um julgamento que não seja imparcial por parte do avaliador. Snell et al. (2015) acrescentam que outro fator negativo desse tipo de avaliação é que, em equipes maiores, o superior hierárquico imediato assume várias funções, o que não permite que ele preste atenção em todos os funcionários de forma a garantir justiça no julgamento.

As sugestões de Snell et al. (2015) corroboram o estudo de Taticchi et al. (2012), que identificaram os marcos presentes em um sistema adequado de gestão de recursos humanos, entre os quais se destacam as considerações das diferentes perspectivas das partes interessadas. Segundo esses autores, é importante que os sistemas de gestão de desempenho de uma organização levem em consideração as perspectivas de seus colaboradores.

Em um primeiro momento, propõe-se que seja adotada, em toda a organização, a autoavaliação dos empregados que, segundo Snell et al. (2015), contribui para que o empregado reflita sobre seus pontos fortes e fracos. Em seguida, propõe-se a formação de Grupo de Trabalho para estudar a implantação de novos sistemas de avaliação, como 
- 360 $0^{\circ}$. Após a adequação estrutural proposta na seção anterior, sugere-se a realização de benchmarking (Leeuw \& Van Den Berg, 2011) em organizações públicas que tenham características similares de tamanho, estrutura e dispersão geográfica de suas unidades.

\subsection{FALTA DE TREINAMENTO DE AVALIADORES E SUBORDINADOS PARA MELHORIA CONTÍNUA DA AVALIAÇÃO DE DESEMPENHO}

Sikora e Ferris (2014) afirmam que, normalmente, são os gerentes de linha os responsáveis pelas práticas de GERH na organização e que o nível de qualidade na implementação destas tem relação positiva com o clima organizacional, a satisfação dos empregados e os seus desempenhos, e relação negativa com a intenção de turnover do empregado. Portanto, se as práticas de GERH são bem implementadas, os resultados operacionais são incrementados e reduz-se o risco de perder empregados de al to desempenho para o mercado de trabalho privado ou para outra organização pública onde o empregado se sinta mais bem valorizado.

Conforme citado anteriormente, as avaliações são de responsabilidade dos líderes imediatos nas organizações públicas brasileiras, ou seja, os gerentes de linha são também avaliadores e devem saber utilizar a avaliação de desempenho como estratégia para potencializar a melhoria contínua. Considerando que a gestão do desempenho é um processo crítico para as organizações, e que menos de um terço dos empregados costumam acreditar que o processo de avaliação ajuda a incrementar seus desempenhos (Gruman \& Saks, 2011), para que ocorra melhoria contínua das avaliações de desempenho dentro das organizações públicas é necessário implementar práticas de treinamentos para todos os colaboradores envolvidos, com o objetivo de melhorar o desempenho do empregado, a assertividade dos instrumentos de avaliação e a atuação do gestor.

Segundo Aguinis e Bradley (2015), é importante identificar e treinar indivíduos dos quais o desempenho é primordial para os resultados estratégicos da empresa. Certamente, os avaliadores se enquadram nesse tipo de indivíduos. Nesse caso, concluiu-se que treinamentos devem fazer parte da rotina de todos os colaboradores da organização, principalmente dos avaliadores (gestores), para reduzir ao máximo a possibilidade de insucesso na implementação de melhorias necessárias à implementação de uma Gestão Estratégica de Recursos Humanos. 


\subsection{RELAÇÃO ENTRE AS AVALIAÇÕES DE DESEMPENHO, O CLIMA ORGANIZACIONAL E A SATISFAÇÃO DOS EMPREGADOS}

Se analisarmos conjuntamente os trabalhos de Russell et al. (2016) e Livi, Alessandri, Caprara, \& Pierro (2015), conclui-se que podem implicar melhoria do clima organizacional e melhora do desempenho de empregados com baixo rendimento, além de refletir na possibilidade de diminuição de turnover, especialmente dos profissionais de alto desempenho.

Livi et al. (2015) identificaram em seus estudos que o positivismo dos empregados contribui para o seu desempenho individual. Para além disso, os autores também identificaram que pessoas com baixo positivismo melhoram seu desempenho quando participam de equipes que possuem pessoas com alto positivismo. Russell et al. (2016) por sua vez, destacam como tem aumentado o número de empregados que são subutilizados dentro das organizações. Esses autores sugerem que, quando as organizações oferecem oportunidades para os empregados superqualificados se desenvolverem na carreira (como lideranças informais ou atividades de mentoring, por exemplo), esses profissionais aproveitarão essas oportunidades e utilizarão suas habilidades, qualidades e experiência para dar contribuições únicas à organização, proporcionando recursos humanos valorizados para a empresa. Segundo Russell et al. (2016), ao dar essas oportunidades (e desafios) aos empregados mais bem qualificados, a empresa proporciona resultados positivos para os empregados (maior satisfação no trabalho e melhora na reputação com relação aos outros empregados) e para a própria organização.

Dessa forma, ao identificar empregados com histórico de alto desempenho, insatisfeitos e desmotivados, e com o objetivo de reduzir o risco de perder empregados de alto desempenho para o mercado, a organização poderá tomar medidas, quais sejam:

a) transferi-lo para uma equipe com empregados de positivismo elevado, contribuindo para elevar também o seu positivismo (Livi et al., 2015);

b) proporcionar oportunidade de mentoring em alguma equipe para que esse empregado de alto desempenho potencialize os resultados da equipe, aumentando com isso sua motivação e satisfação no trabalho (Russell et al., 2016) $e$, consequentemente, fazer com que permaneça engajado com seu trabalho $e$ com a organização (Gruman \& Saks, 2011; Vosloban, 2012). 


\section{CONCLUSÃO}

A presente pesquisa foi realizada com o objetivo de analisar o desempenho individual e a percepção do cumprimento da missão organizacional pelos trabalhadores de uma empresa pública brasileira. $\mathrm{O}$ baixo resultado do $\mathrm{R}^{2}$ confirmou a necessidade de pesquisar outras variáveis que impactam a percepção do cumprimento da missão institucional pelos trabalhadores públicos da empresa brasileira estudada.

Com este estudo se pode elencar possíveis soluções para o processo de GERH em uma empresa estatal brasileira de grande porte, baseado em uma revisão de literatura sobre gestão de pessoas e o impacto das práticas de treinamento, recrutamento e seleção, recompensa e avaliação na percepção, satisfação e produtividade do trabalhador.

De acordo com o estudo realizado, apesar da constatação de que é um grande desafio a implantação de um modelo com práticas de GERH, foram identificadas possibilidades de reflexão e implementação de melhorias explicitadas pelas discussões na quarta seção, que servirão tanto para a empresa estudada quanto para o funcionalismo público brasileiro em geral. Entretanto, por se tratar de uma empresa formada exclusivamente por capital público, dependerá de vontade política para a implementação de possíveis melhorias propostas nas discussões.

A pesquisa contribui para demonstrar que os temas GERH e GD são um desafio comum e objeto de pesquisa em vários países e em várias organizações públicas e privadas. Ainda não há resposta para todas as questões de forma simples e objetiva, mas, com esta pesquisa, percebe-se que a solução para os problemas enfrentados pelas organizações no processo de gestão e avaliação de desempenho dos empregados pode estar na produção científica com estudos de aplicabilidade prática sobre o tema.

Considerando a Avaliação e Gestão do Desempenho do trabalhador, segundo Yadav e Dabhade (2013), as avaliações, em um ciclo de avaliação de desempenho, são normalmente realizadas duas vezes por ano em uma organização, sob a forma de revisões médias e avaliações anuais que são realizadas no final do exercício financeiro. Nesse processo, o avaliador primeiro oferece as avaliações autopreenchidas no formulário de autoavaliação e também descreve suas realizações ao longo de um período em termos quantificáveis. Após a autoavaliação, as avaliações finais devem ser fornecidas pelo avaliador com as conquistas quantificáveis e mensuráveis do empregado sendo avaliado. Todo o processo de revisão busca uma participação ativa, tanto do empregado quanto do avaliador, para analisar as causas das falhas no desempenho e como isso pode ser superado, e, isso deve ser discutido em uma reunião de feedback de desempenho.

Assim, propôs-se uma análise crítica da Gestão e Avaliação de Desempenho dos colaboradores da organização estudada e uma revisão dos processos de treinamento, 
recrutamento e seleção, recompensa e avaliação com foco na percepção do trabalhador e nas necessidades da organização em aumentar a eficiência e a eficácia de seus processos.

Com relação à recompensa, sabe-se das dificuldades de remuneração variável el ou gratificações por produtividade para o setor público, mas entende-se serem possíveis premiações pelas metas superadas ou por projetos realizados dentro de um cronograma proposto.

Finalmente, compreende-se que, por se tratar de uma empresa estatal e por cumprimento da legislação inerente ao serviço público brasileiro, não é possível recrutar funcionários sem concurso público, mas considera-se ser necessária uma revisão de critérios para a ascensão na carreira com objetividade, clareza e transparência para que o empregado se sinta motivado a melhorar e aumente sua confiança na organização, aumentando também sua satisfação e seu desempenho. Assim, conclui-se que a percepção do cumprimento da missão organizacional pelos trabalhadores da empresa pública estudada é influenciada por fatores endógenos e exógenos à organização e intrínsecos e extrínsecos ao empregado, visto que fatores intrínsecos e endógenos obtiveram maior relevância na alteração de percepção do trabalhador.

\section{REFERÊNCIAS}

Aguinis, H., \& Bradley, K. J. (2015). The secret sauce for organizational success: Managing and producing star performers. Organizational Dynamics, 44(3), 161-168.

Akim, É. K., \& Mergulhão, R. C. (2015). Panorama da produção intelectual sobre a medição de desempenho na gestão pública. Revista de Administração Pública, 49(2), 337-366.

Arellano-Gault, D., Demortain, D., Rouillard, C., \& Thoenig, J. C. (2013). Bringing public organization and organizing back in. Organization Studies, 34(2), 145-167.

Assis, L. O. M. de, \& Reis, M. T., Neto. (2011). Remuneração variável no setor público: Investigação das causas do fracasso e implicações para o Estado Brasileiro. Gestão. Org., 9(3), 585-614.

Bedarkar, M., \& Pandita, D. (2014). A study on the drivers of employee engagement impacting employee performance. Procedia-Social and Behavioral Sciences, 133, 106-115.

Bronkhorst, B., Steijn, B., \& Vermeeren, B. (2015). Transformational leadership, goal setting, and work motivation: The case of a Dutch municipality. Review of Public Personnel Administration, 35(2), 124-145. 
Campbell, J. W., \& Im, T. (2016). PSM and turnover intention in public organizations: Does change-oriented organizational citizenship behavior play a role? Review of Public Personnel Administration, 36(4), 323-346.

Currie, G., \& Procter, S. J. (2005). The antecedents of middle managers' strategic contribution: The case of a professional bureaucracy. Journal of management studies, 42(7), 1325-1356.

Dutra, J. S. (2002). Gestão de pessoas: Modelo, processos, tendências e perspectivas. Rio de Janeiro: Atlas.

Ford, R. C., Latham, G. P., \& Lennox, G. (2011). Mystery shoppers. Organizational Dynamics, 3(40), 157-164.

Gabelica, C., Van Den Bossche, P., De Maeyer, S., Segers, M., \& Gijselaers, W. (2014). The effect of team feedback and guided reflexivity on team performance change. Learning and Instruction, 34, 86-96.

Gruman, J. A., \& Saks, A. M. (2011). Performance management and employee engagement. Human Resource Management Review, 21(2), 123-136.

Hair, F., Jr., Black, W. C., Babin, B. J., Anderson, R. E., \& Tatham, R. L. (2009). Análise multivariada de dados. Porto Alegre: Bookman Editora.

Klein, F. A., \& Mascarenhas, A. O. (2016). Motivação, satisfação profissional e evasão no serviço público: $O$ caso da carreira de especialistas em Políticas Públicas e Gestão Governamental. Revista de Administração Pública, 50(1), 17-39.

Leeuw, S. de, \& Van Den Berg, J. P. (2011). Improving operational performance by influencing shopfloor behavior via performance management practices. Journal of Operations Management, 29(3), 224-235.

Lim, S., Wang, T. K., \& Lee, S. Y. (2017). Shedding new light on strategic human resource management: The impact of human resource management practices and human resources on the perception of federal agency mission accomplishment. Public Personnel Management, 46(2), 91-117.

Livi, S., Alessandri, G., Caprara, G. V., \& Pierro, A. (2015). Positivity within teamwork: Cross-level effects of positivity on performance. Personality and Individual Differences, $85,230-235$.

Lotta, G. S. (2002). Avaliação de desempenho na área pública: Perspectivas e propostas frente a dois casos práticos. RAE-eletrônica, 1(2), 1-12. 
Lugoboni, L. F., Cabral, C. S. C., Chirotto, A. R., \& Zittei, M. V. M. (2018). Indicadores de desempenho como ferramenta de gestão no terceiro setor: Um caso prático aplicado a um sindicato patronal. Race, Revista de Administração, Contabilidade e Economia, 17(2), 733-756.

Neely, A., Gregory, M., \& Platts, K. (2005). Performance measurement system design: A literature review and research agenda. International journal of operations \& production management, 25(12), 1228-1263.

Ojokuku, R., \& Sajuyigbe, A. S. (2014). Effect of employee participation in decision making on performance of selected small and medium scale enterprises in lagos, Nigeria. European Journal of Business and Management, 93.

Oliveira Nogueira, M. D. L. de, Costa, L. V., \& Santos Claro, J. A. C. dos. (2015). Relação entre estilo de liderança e comprometimento organizacional afetivo. Race, Revista de Administração, Contabilidade e Economia, 14(2), 707-736.

Russell, Z. A., Ferris, G. R., Thompson, K. W., \& Sikora, D. M. (2016). Overqualified human resources, career development experiences, and work outcomes: Leveraging an underutilized resource with political skill. Human Resource Management Review, 26(2), 125-135.

Sikora, D. M., \& Ferris, G. R. (2014). Strategic human resource practice implementation: The critical role of line management. Human Resource Management Review, 24(3), 271-281.

Snell, S., Morris, S., \& Bohlander, G. W. (2015). Managing human resources. Scarborough, Canada: Nelson Education.

Taticchi, P., Balachandran, K., \& Tonelli, F. (2012). Performance measurement and management systems: State of the art, guidelines for design and challenges. Measuring Business Excellence, 16(2), 41-54.

Vosloban, R. I. (2012). The Influence of the Employee's Performance on the company's growth-a managerial perspective. Procedia economics and finance, 3, 660-665.

Yadav, R. K., \& Dabhade, N. (2013). Performance management system in Maharatna Companies (a leading public sector undertaking) of India-a case study of BHEL, Bhopal (MP). International Letters of Social and Humanistic Sciences, 4(49), 49-69.

Yu, Y., Dong, X. Y., Shen, K. N., Khalifa, M., \& Hao, J. X. (2013). Strategies, technologies, and organizational learning for developing organizational innovativeness in emerging economies. Journal of Business Research, 66(12), 2507-2514. 


\section{Como citar este artigo:}

\section{ABNT}

Araujo, Uerlei Valdomiro et al. Percepção do cumprimento da missão organizacional e desempenho individual em uma empresa pública brasileira. RACE, Revista de Administração, Contabilidade e Economia, Joaçaba: Editora Unoesc, v. 18, n. 2, p. 321-344, maio/ ago. 2019. Disponível em: http://editora.unoesc.edu.br/index.php/race. Acesso em: dia/mês/ ano.

\section{APA}

Araujo, V. A, Baldam, R. de L., Coelho, T. de P., Jr., \& Costa, L. (2019). Percepção do cumprimento da missão organizacional e desempenho individual em uma empresa pública brasileira. RACE, Revista de Administração, Contabilidade e Economia, 18(2), 321-344. Recuperado de http://editora.unoesc.edu.br/index.php/race 